\title{
PAPER
}

\section{Donepezil for cognitive impairment in Parkinson's disease: a randomised controlled study}

\author{
D Aarsland, K Laake, J P Larsen, C Janvin
}

J Neurol Neurosurg Psychiatry 2002;72:708-712

See end of article for authors' affiliations

......................

Correspondence to: Dr D Aarsland, Section of Geriatric Psychiatry, Psychiatric Hospital of Rogaland, Arm. Hansen v 20, 4095 Stavanger Norway;

aarsland@netpower.no

Received 9 August 2001 In revised form

19 November 2001

Accepted

14 December 2001 inesterase inhibitor donepezil in patients with Objective: To study the safety and efficacy of the
Parkinson's disease (PD) and cognitive impairment.

Methods: This was a double blind, randomised and placebo controlled, crossover study in which 14 patients with PD and cognitive impairment received donepezil (5 or $10 \mathrm{mg}$ per day) or matching placebo during two sequential periods lasting 10 weeks each. The primary outcome measures were the mini mental state examination (MMSE) score, the clinician's interview based impression of change plus caregiver input $(\mathrm{ClBIC}+)$ score, and the motor subscale of the unified Parkinson's disease rating scale (UPDRS).

Results: Two patients on donepezil (14\%) dropped out after one and four weeks of the first treatment period because of peripheral cholinergic side effects, otherwise the adverse effects were few and not severe. Carryover or residual effects were not observed. Parkinsonism did not increase during donepezil treatment. After 10 weeks of treatment, the mean MMSE score was increased by 2.1 (SD 2.7) points on donepezil and 0.3 (SD 3.2) points on placebo, and the CIBIC+ score was 3.3 (SD 0.9) on donepezil and 4.1 (SD 0.8) on placebo. Statistical analysis of the repeated measurements and crossover study design showed significant effects of donepezil compared with placebo for MMSE $(p=0.013)$ and $\mathrm{CIBIC}+(p=0.034)$. Five $(42 \%)$ patients on donepezil and two $(17 \%)$ on placebo were rated as improved on the basis of the $\mathrm{CIBIC}+$ score.

Conclusions: Donepezil improves cognition, and seems to be well tolerated and not to worsen parkinsonism in patients with cognitive impairment.
$\mathrm{T}$ he prevalence of Parkinson's disease (PD) is nearly $1 \%$ in the population 65 years of age and above. ${ }^{1}$ Cognitive impairment and dementia often accompany the motor manifestations of the disease. Studies suggest that $25-30 \%$ of patients with PD are demented, ${ }^{2}$ and the risk of dementia in $\mathrm{PD}$ is nearly six times higher than in the general population. ${ }^{3}$ Cognitive impairment in patients with PD has important clinical consequences with regard to risk for nursing home placement $^{4}$ and caregiver stress. ${ }^{5}$

PD is caused by a progressive degeneration of dopaminergic neurones in the substantia nigra where the principal neuropathological finding is Lewy bodies. ${ }^{6}$ These are also found in the cortex of most patients with $\mathrm{PD}^{7}$ and may contribute to the development of dementia. ${ }^{8}$ Neuropathological ${ }^{9}$ and imaging ${ }^{10}$ studies in patients with PD who have dementia have shown appreciable dysfunction of the cholinergic neurones in the nucleus of Meynert and their cortical terminals, suggesting that cholinergic deficits are also involved in the cognitive impairment in PD.

Cholinergic deficits are typical in Alzheimer's disease, ${ }^{9}$ in which treatment with cholinesterase inhibitors improves cognition and activities of daily living. ${ }^{11}$ There is also evidence to suggest that cholinergic agents improve cognition in dementia with Lewy bodies (DLB), ${ }^{12}$ a disorder with clinical, neurochemical, and pathological similarities to PD. ${ }^{13}$ Preliminary results from an open study of treatment of cognitively impaired patients with PD with the cholinesterase inhibitor tacrine ${ }^{14}$ suggest that this class of drugs improves cognition in PD, but placebo controlled studies have not yet been reported. The aim of this study was therefore to test the hypothesis that a cholinesterase inhibitor, donepezil, improves cognition in patients with PD and cognitive impairment. Because concern has been expressed that cholinesterase inhibitors may increase parkinsonism, the motor symptoms of PD were monitored during the study.

\section{MATERIALS AND METHODS}

Consecutive patients with PD and cognitive impairment, diagnosed at the outpatient clinic of the Department of Neurology at the Central Hospital of Rogaland, Stavanger, were invited to participate. The diagnostic evaluation comprised physical and neurological examinations, a clinical interview of the patient and caregiver based on the Diagnostic and statistical manual of mental disorders, 4th ed ${ }^{15}$ (DSM-IV) criteria for dementia due to $\mathrm{PD}$, neuropsychological examination, routine blood tests, a computed tomography scan of the brain, and a chest radiograph. To exclude patients with a lower probability of idiopathic $\mathrm{PD}$, only patients with clinically definite and probable PD, according to published criteria, ${ }^{16}$ were recruited to the study. A diagnosis of clinically definite PD requires that the patient has resting tremor, at least two additional cardinal signs (akinesia, rigidity, or postural abnormalities), unilateral onset and asymmetrical development of the disease, and a good to excellent response to a dopaminergic drug. No atypical features should be present in the history or on the clinical examination. For a diagnosis of clinically probable PD, the patient should have at least two of the four cardinal features of parkinsonism. Resting tremor is not mandatory, and not more than one of the following clinical features may be present: mild dementia or clinically relevant autonomic failure at disease onset; symmetrical disease presentation; no better than a moderate response to a dopaminergic agent; other atypical features.

The inclusion criteria were: mild to severe parkinsonism (Hoehn and Yahr ${ }^{17}$ stage less than 5); age 45-95 years; clinical

Abbreviations: PD, Parkinson's disease; MMSE, mini mental state examination; UPDRS, unified Parkinson's disease rating scale; NPI, neuropsychiatric inventory; DLB, dementia with Lewy bodies 
evidence of decline in memory and at least one other category of cognitive function; onset of cognitive decline starting one year or more after the onset of parkinsonism; a mini mental state examination (MMSE) ${ }^{18}$ score of 16-26. (Patients with more severe cognitive impairment would not be able to complete the neuropsychological battery.) The treatment of the disease should have remained stable during the month preceding the baseline evaluation, and was continued during the study. The spouse or another caregiver accompanied the patient to the hospital for the assessments to act as an informant. Patients with brain disease other than PD or with other severe medical disorders were excluded. The subjects were not be taking anticholinergic drugs or psychotropic agents with anticholinergic effects. All patients provided informed consent, and the study protocol was approved by the committee for ethics in biomedical research of the University of Bergen, Norway.

\section{Design}

This was a double blind, placebo controlled, crossover trial with two treatment periods of 10 weeks each. A randomisation list was computer generated according to a randomised block design. The various treatment-placebo and placebotreatment blocks were then issued with a medication number and assigned to consecutive patients. The principal investigator (DA) was given a sealed envelope containing the individual treatment regimens of each patient. This envelope was to be opened only in case of emergency. All personnel involved in the study remained unaware of the group assignment until all patients had completed the trial.

The assessments took place at baseline and after six and 10 weeks of each treatment period-that is, at week 6, 10, 16, and 20. A previous study of donepezil in patients with Alzheimer's disease showed that cognition declined to baseline level within three weeks of withdrawal. ${ }^{19}$ A washout period between the two treatment periods was therefore not considered necessary. The initial dose was donepezil $5 \mathrm{mg}$ or identically appearing placebo tablets taken once a day in the evening. The dose was increased to $10 \mathrm{mg}$ after six weeks if well tolerated. Glasses containing 49 or 35 tablets were given to the patient and caregiver for each six or four week treatment period respectively - that is, one glass for each patient and each dose. The assessments were scheduled at about the same time of the day for each patient, and patients with parkinsonian motor fluctuations were examined in their "on phase" - that is, at their best level of motor functioning. Short acting hypnotics (zolpidem or zopiklon) were allowed, but longer acting benzodiazepines were not allowed to be taken within the 24 hours before testing.

\section{Outcome measures}

The primary outcomes were: $(a)$ the MMSE score; $(b)$ the clinician's interview based impression of change (CIBIC+), which provides a global impression of a patient's cognitive improvement or deterioration over time, scored on the basis of an interview with the patient and a caregiver on a seven point scale $(\mathrm{l}=$ very much improved; $4=$ no change; $7=$ very much worse) by a clinician, blinded to other assessments; (c) the motor subscale of the unified Parkinson's disease rating scale (UPDRS), ${ }^{20}$ which assesses the severity of parkinsonism. In addition, psychiatric symptoms such as hallucinations, delusions, agitation, depression, and apathy were rated by the psychiatrist using the neuropsychiatric inventory (NPI) ${ }^{21}$ The caregiver and patient also evaluated whether parkinsonism had improved, declined, or remained unchanged during each treatment period using the same seven point scale as the CIBIC+. In addition, the patients completed a battery of neuropsychological tests assessing verbal and visual memory, executive functions, visuospatial abilities, and attention. These results will be described in more detail elsewhere.

\section{Drug compliance and safety}

Dosing compliance was assessed by interviewing the caregiver and tablet counts. Patients were excluded if the number of tablets removed from the bottles divided by the number of treatment days between each visit was outside the range 70-130\%. Safety evaluations conducted at each assessment point comprised vital signs and an interview based check list for adverse events based on the Scandinavian society for psychopharmacological scale for side effects. ${ }^{22}$

\section{Statistical analysis}

Detailed analysis of the outcome variables were carried out using the general linear model (SYSTAT, SPSS Inc) to encompass the repeated measurement and crossover structure of the design. Subject, treatment, period, and a residual effect (nested with period) of the treatment given in the previous period (coded as none, drug, or placebo) were modelled as categorical. Tests for carryover and period effects were performed. Patients were included in the efficacy analysis if they had both a baseline score and at least one score after the baseline (last observation carried forward). Differences in frequency of side effects were tested with McNemar's test of symmetry using exact methods. Data from patients who had received at least one dose of study drugs were considered for the safety analysis. $\mathrm{p}<0.05$ was assumed to be significant.

\section{RESULTS}

Thirty three patients were screened, and 14 (13 men, one woman) were randomised (fig 1). Two patients (14\%), both male, withdrew from the study because of adverse reactions (dizziness, nausea, and diarrhoea) before the evaluation at week 6 of the first study period; both were receiving donepezil. The remaining 12 all completed the study, assessed according to the protocol, and included in the further analyses. Dose increments were carried out according to the protocol for 11 of the patients. In one patient, the dose was not increased owing to a misunderstanding, and this patient continued on $5 \mathrm{mg}$ donepezil during the final four weeks of the treatment period. Tablet counts showed a high degree of drug compliance (mean 101\%, range $83-123 \%$ ).

Seven subjects were diagnosed as suffering from clinically definite PD and five from clinically probable PD. The mean Hoehn and Yahr stage was 2.4 (range 1-4), age 71.0 (SD 3.9) years, duration of PD 10.8 (SD 5.2) years, baseline MMSE score 20.8 (SD 3.4) (range 16-26), duration of cognitive decline 3.0 ( SD 2.6) years, and levodopa dose 485 (SD 256) mg a day. The two treatment groups did not differ with regard to baseline MMSE score, age, or duration of disease. There was a trend towards higher mean UPDRS motor score in the donepezil-placebo group (35.3 (SD 17.0)) compared with the placebo-donepezil group $(28.8(\mathrm{SD} 14.0)) \quad(\mathrm{t}=0.7, \mathrm{df}=10$, $\mathrm{p}=0.49$ ). Eleven patients had impairment of at least two cognitive domains other than memory. Eight patients fulfilled all the DSM-IV criteria for dementia due to PD, while significant functional impairment due to cognitive deficits could not be definitely verified for four.

\section{Effects on primary outcomes}

At the end of both treatment periods, patients on donepezil had higher MMSE and lower CIBIC+ scores than those on placebo (table 1), indicating improvement under donepezil treatment. The statistical modelling of the MMSE and the CIBIC + data showed no significant residual effect nested with treatment period $(p=0.87$ and $p=0.53$ respectively). In the group receiving donepezil first, the MMSE score six weeks after crossover to placebo was 20.3 (SD 3.2), compared with 20.8 (SD 3.9) at baseline. There was thus no indication of a carryover effect. A significant treatment effect of donepezil on the MMSE score compared with placebo was found $(F=9.1$, 


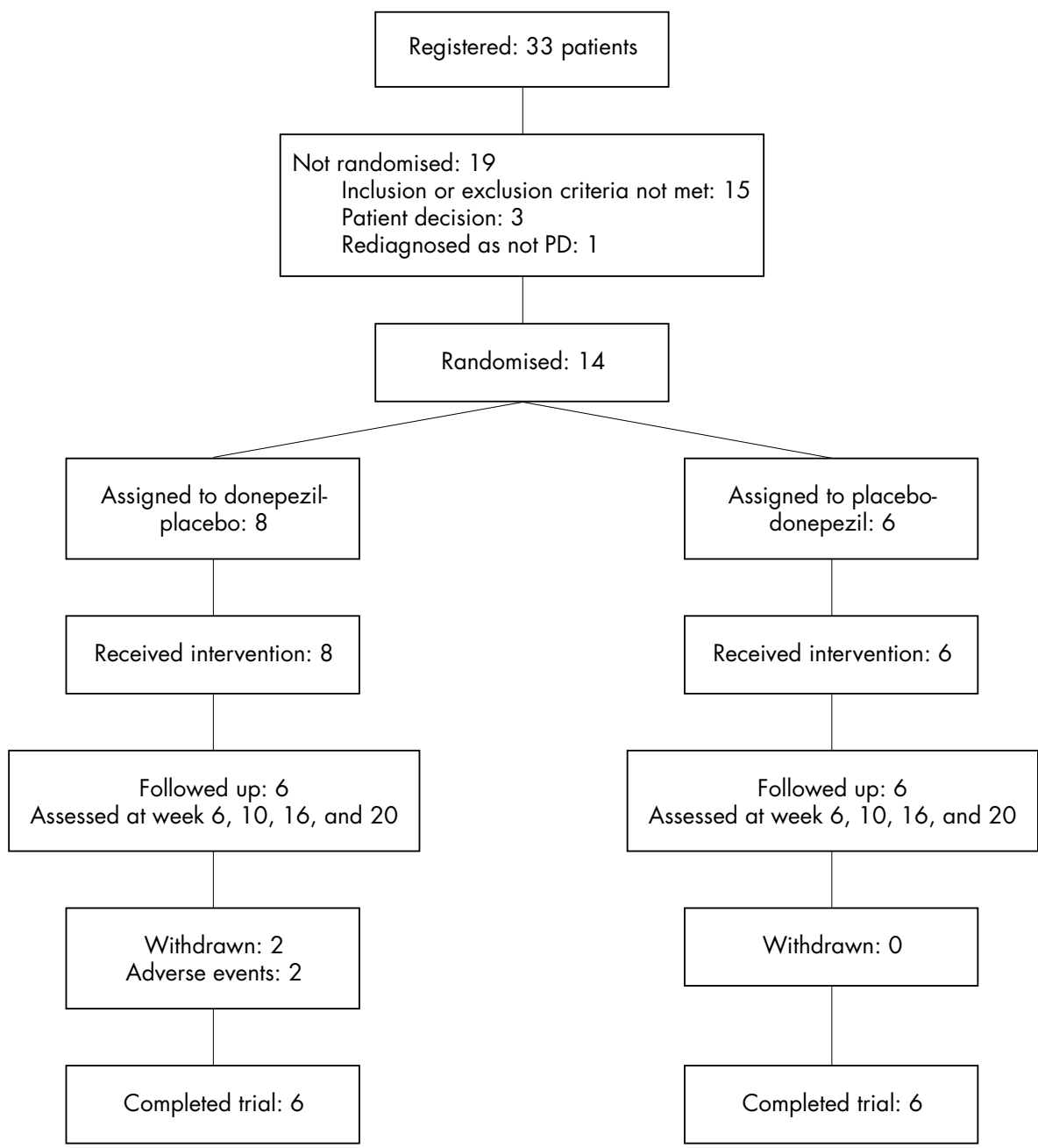

Figure 1 Progress of the patients throughout the 20 weeks of the crossover trial. Intervention was the administration of donepezil or placebo during two treatment sequences of 10 weeks each.

$\mathrm{df}=1, \mathrm{p}=0.013)$. On donepezil, the mean MMSE score difference from baseline to week 10 was 2.1 (SD 2.7) points and on placebo 0.3 (SD 3.2) points. Figure 2 shows the mean MMSE scores by group. There were negative, but non-significant, correlations between baseline MMSE scores and change during donepezil treatment at week 6 (Pearson $r=-0.01$ ) and week 10 $(r=-0.38, \mathrm{p}=0.22)$.

Three patients had missing data on the CIBIC+ (two at week 6 and one at week 16). These data points were coded as no change. After 10 weeks of treatment, five $(42 \%)$ patients on donepezil and two ( $17 \%)$ on placebo were rated as improvedthat is, a CIBIC + score of 3 or lower. The mean CIBIC + score at week 10 was 3.3 (SD 0.9) during donepezil treatment and
4.1 (SD 0.8) during placebo treatment. Statistical modelling showed a significant treatment effect in favour of donepezil on this outcome also $(F=6.0, \mathrm{df}=1, \mathrm{p}=0.034)$. Reanalysis of the data without recoding the missing CIBIC+ data points, thereby excluding data from three cases from the analysis, resulted in a p value of 0.079 .

The scores on the UPDRS motor subscale disclosed no deterioration in parkinsonian symptoms after 10 weeks of treatment with donepezil $(p=0.37)$. Similarly, neither the caregivers nor the patients reported worsening of parkinsonism $(p=0.20)$ (table 1$)$. Few of the 12 patients had positive NPI scores on delusions $(n=3)$, hallucinations $(n=2)$, agitation $(n=1)$, depression $(n=6)$, or apathy $(n=5)$ at baseline, and

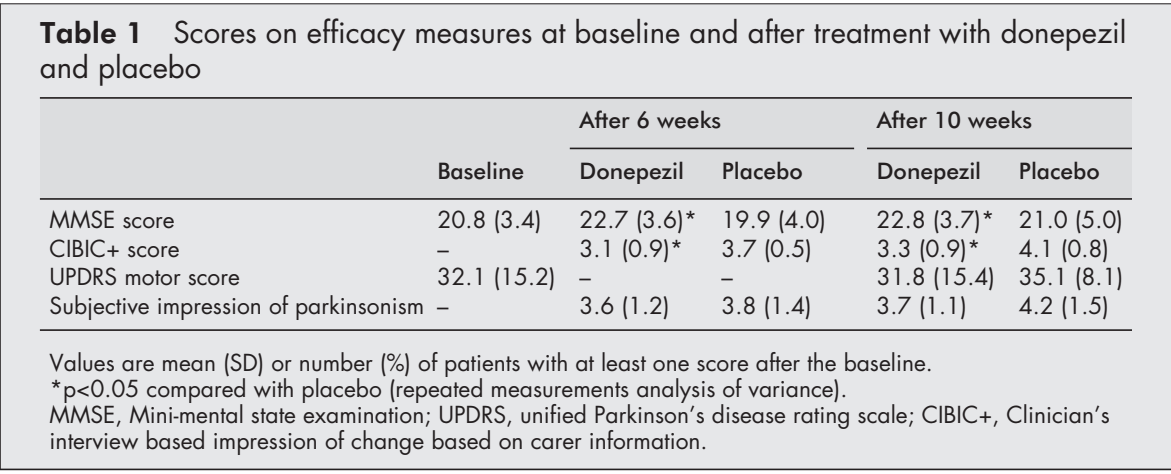




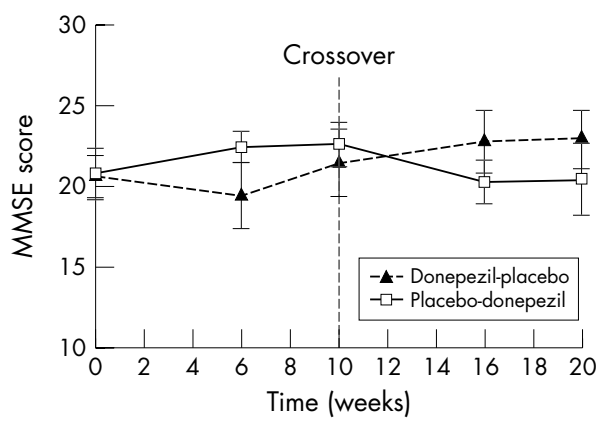

Figure 2 Change in mini mental state examination (MMSE) score from baseline over the two treatment sequences. Values are mean (SE).

Table 2 Number with adverse events reported during treatment with donepezil and placebo

\begin{tabular}{lll}
\hline & \multicolumn{2}{l}{$\begin{array}{l}\text { Any degree } \\
\text { (moderate/severe) }\end{array}$} \\
\cline { 2 - 3 } & $\begin{array}{l}\text { Donepezil } \\
\left(n=14^{*}\right)\end{array}$ & $\begin{array}{l}\text { Placebo } \\
(n=12)\end{array}$ \\
\hline Nausea & $4(3)$ & $2(1)$ \\
Headache & $4(3)$ & $3(1)$ \\
Tiredness & $7(6)$ & $6(5)$ \\
Insomnia & $2(1)$ & $2(1)$ \\
Increased dreaming & $3(2)$ & $5(2)$ \\
Dizziness & $7(3)$ & $3(1)$ \\
Increased sweating & $6(5)$ & $3(2)$ \\
Increased salivation & $6(4)$ & $4(1)$ \\
Dry mouth & $4(2)$ & $5(2)$ \\
Diarrhoea & $2(2)$ & $2(1)$ \\
Constipation & $5(2)$ & $4(0)$ \\
Urinary retention & 0 & 0 \\
Rash & $3(0)$ & 0 \\
Any adverse event & $12(11)$ & $9(7)$ \\
Number of adverse events & & \\
per person, mean (SD) & $4.2(3.2)$ & $2.8(1.0)$ \\
\hline * 14 patients received donepezil treatment, but \\
only 12 received placebo treatment because two \\
patients dropped out. & & \\
\hline
\end{tabular}

mean NPI scores were low at baseline. No significant treatment effects were observed with regard to any of the NPI items (results not shown).

\section{Adverse events}

Table 2 shows the proportion of patients reporting adverse events. The number of patients reporting any adverse events while taking donepezil was 10 out of $14(71 \%)$ and while taking placebo nine out of 12 (75\%) (14 patients entered the donepezil condition, and only 12 the placebo condition). Most specific examples of adverse events were more common during donepezil than during placebo treatment, but statistical significance (McNemar's test) was not reached. However, owing to the few patients in the study, the power of detecting significant differences was low.

\section{DISCUSSION}

This study shows that donepezil improves performance on the MMSE and the clinician's interview based impression of cognition in patients with PD and cognitive impairment. Clinical improvement was reported for $42 \%$ of the patients receiving donepezil compared with $17 \%$ receiving placebo. To the best of our knowledge, this is the first placebo controlled trial reporting a benefit of a cholinesterase inhibitor in patients with PD and cognitive impairment. We found no indication that such treatment worsens parkinsonism. Two of 14 patients withdrew because of typical side effects of donepezil. The remaining 12 did not report significantly more side effects in the donepezil than the placebo condition. However, the power of this small study is evidently too low to give precise estimates for the prevalence of side effects of donepezil in patients with PD and cognitive impairment

The small sample size and the skewed sex distribution may raise concern about the representativeness of the patients. However, age, disease duration, stage of parkinsonism, and level of cognitive impairment as measured by the MMSE score were similar to that of a representative community based sample of patients with PD and mild to moderate dementia previously reported. ${ }^{2}$ Eight patients fulfilled the DSM-IV criteria for dementia due to PD, and another three fulfilled the dementia criteria suggested by Cummings and Benson. ${ }^{23}$ This suggests that the results are generalisable to patients with PD and mild to moderate dementia.

As patients with parkinsonism and dementia may also fulfil criteria for possible DLB, ${ }^{24}$ there was a possibility that patients with DLB erroneously diagnosed as suffering from PD were included. Care was therefore taken to exclude patients who might fulfil clinical criteria for DLB. According to an international panel of experts, the main clinical feature distinguishing PD from DLB is the sequence of the development of symptoms. In particular, if dementia ${ }^{24}$ or hallucinations ${ }^{25}$ develops within one year of onset of parkinsonism, such patients should be diagnosed with DLB and not PD. This type of patient was excluded from this study. In our study, the mean duration of parkinsonism was nearly 10 years before cognitive decline started to become evident, strongly indicating that the patients included would have been diagnosed as PD and not DLB using modern diagnostic criteria. ${ }^{26}$ On the other hand, patients with PD often have some Lewy bodies in their cerebral cortex ${ }^{7}$ and recent studies suggest that cortical Lewy bodies contribute to dementia in PD. ${ }^{8}$ Thus, DLB and PD with dementia may be neuropathologically indistinguishable. Further studies are needed to clarify the exact clinical, pathological, and nosological distinction between DLB and PD.

The advantage of a crossover design is that comparison can be made within subjects rather than between subjects, and the sample size needed will therefore be smaller. ${ }^{27}$ However, the disadvantage is the risk of confounding caused by carryover and period effects, and that patients may be withdrawn during the first treatment period and therefore not receive the second treatment. The results in our study did not indicate any carryover effect. This is consistent with studies on Alzheimer's disease showing that the effect of donepezil had disappeared three weeks after withdrawal. ${ }^{19}$

Neocortical cholinergic activity is reduced in PD and at least as severe in patients with PD and dementia as in patients with Alzheimer's disease. ${ }^{928}$ Choline acetyltransferase activity in the temporal cortex of patients with PD and dementia correlates with the number of cholinergic neurones in the nucleus of Meynert and with cognition, but not with the extent of plaques or tangles. ${ }^{29}$ There are indications that postsynaptic muscarinic receptors are better preserved and are more functionally intact in patients with PD and dementia than in those with Alzheimer's disease. ${ }^{9}$ Accordingly, cholinergic drugs may be more effective in demented patients with PD than in those with Alzheimer's disease. This may explain why the differences between active drug and placebo on the MMSE and CIBIC + scores seemed more pronounced in our study than in trials on patients with Alzheimer's disease. ${ }^{11}$ This is also in line with previous reports suggesting a better response to cholinergic treatment in DLB than in Alzheimer's disease. ${ }^{30}$ However, given the crossover design and the limited number of patients, our findings must be confirmed in larger parallel group trials before donepezil can be recommended for cognitive impairment in patients with PD. 


\section{ACKNOWLEDGEMENTS}

The study received partial support from Pfizer Norway Inc. We thank N G Lim and H R Marianayagam for assistance with patient assessment.

\section{Authors' affiliations}

D Aarsland, C Janvin, Section of Geriatric Psychiatry, Psychiatric Hospital of Rogaland, Stavanger, Norway

K Laake, Department of Geriatric Medicine, Ullevål Hospital, Oslo, Norway

J P Larsen, Department of Neurology, Central Hospital of Stavanger, Norway

Competing interests: DA, JPL, and KL have been reimbursed by Pfizer Inc, the manufacturer of donepezil, for attending several conferences, and have received fees for speaking, consulting, and organising education. The present study was partially funded by a grant from Pfizer Inc. None of the authors have been employed by, or hold any stocks or shares in, Pfizer.

\section{REFERENCES}

1 Tandberg E, Larsen JP, Nessler EG, et al. The epidemiology of Parkinson's disease in the county of Rogaland, Norway. Mov Disord 1995; 10:541-9.

2 Aarsland D Tandberg E, Larsen JP, et al. Frequency of dementia in Parkinson's disease. Arch Neurol 1996;53:538-42.

3 Aarsland D, Andersen K, Larsen JP, et al. Risk of dementia in Parkinson's disease: a community-based, prospective study. Neurology 2001:56:730-6.

4 Aarsland D, Larsen JP, Karlsen K, et al. Mental symptoms in Parkinson's disease are important contributors to caregiver distress. Int J Geriatr Psychiatry 1999;14:866-74

5 Aarsland D, Larsen JP, Tandberg E, et al. Predictors of nursing home placement in Parkinson's disease: a population-based, prospective study. J Am Geriatr Soc 2000;48:938-42.

6 Forno LS. Neuropathology of Parkinson's disease. J Neuropathol Exp Neurol 1996;55:259-72.

7 Hughes AJ, Daniel SE, Blankson S, et al. A clinicopathologic study of 100 cases of Parkinson's disease. Arch Neurol 1993:50:140-8.

8 Hurtig HI, Trojanowski JQ, Galvin J, et al. Alpha-synuclein cortical lewy bodies correlate with dementia in Parkinson's disease. Neurology 2000;54:1916-21

9 Perry E, Irving D, Kerwin JM, et al. Cholinergic transmitter and neurotrophic activities in Lewy body dementia: similarity to Parkinson's and distinction from Alzheimer disease. Alzheimer Dis Assoc Disord 1993; 7:69-79

10 Kuhl DE, Minoshima S, Fessler JA, et al. In vivo mapping of cholinergic terminals in normal aging, Alzheimer's disease, and Parkinson's disease. Ann Neurol 1996:40:399-410.

11 Rogers SL, Farlow MR, Doody RS, et al. A 24-week

double-blind, placebo-controlled study of donepezil in patients with Alzheimer's disease. Neurology 1998;50:136-45.
12 McKeith I, Del Ser, Spano PF, et al. Efficacy of rivastigmine in dementia with Lewy bodies: a randomised, double-blind placebo-controlled international study. Lancet 2000;356:2031-6.

13 McKeith IG, Burn D. Spectrum of Parkinson's disease, Parkinson's dementia, and Lewy body dementia. Neurol Clin 2000;18:865-83.

14 Hutchinson M, Fazzini E. Cholinesterase inhibition in Parkinson's disease. J Neurol Neurosurg Psychiatry 1996;61:324-6.

15 American Psychiatric Association. Diagnostic and statistical manual of mental disorders. 4th ed. Revised. Washington DC: American Psychiatric Association

16 Larsen JP, Dupont E, Tandberg E. The clinical diagnosis of Parkinson's disease: proposal of diagnostic subgroups classified at different levels of confidence. Acta Neurol Scand 1994;84:242-51.

17 Hoehn MH, Yahr MD. Parkinsonism: onset, progression, and mortality. Neurology 1967; 17:427-42.

18 Folstein MF, Folstein SE, McHugh PR. "Mini-Mental State": a practical method for grading the mental state of patients for the clinician. $J$ Psychiatr Res 1975;12:189-98.

19 Greenberg SM, Tennis MK, Brown LB, et al. Donepezil therapy in clinical practice. A randomized cross-over study. Arch Neurol 2000;57:94-9.

20 Fahn S, Elton RL. Members of the UPDRS Development Committee. Unified Parkinson's Disease Rating Scale. In: Fahn S, Marsden CD, Calne $\mathrm{DB}$, et al, eds. Recent development in Parkinson's disease. Florham Park, NJ: Macmillan Health Care Information, 1987:153-63.

21 Cummings JL, Mega M, Gray K, et al. The Neuropsychiatric Inventory comprehensive assessment of psychopathology in dementia. Neurology 1994:44:2308-14.

22 Lingiærde O, Ahlfors UG, Bech $\mathrm{P}$, et al. The UKU side effect rating scale. A new comprehensive rating scale for psychotropic drugs and a cross-sectional study of side-effects in neuroleptic-treated patients. Acta Psychiatr Scand 1987;76(suppl):334.

23 Cummings JL, Benson DF. Dementia: a clinical approach. 2nd ed. Boston: Butterworth-Heinemann, 1992.

24 McKeith IG, Galasko D, Kosaka K, et al. Consensus guidelines for the clinical diagnosis of dementia with Lewy bodies (DLB): report of the consortium on DLB international workshop. Neurology 1996;47:1113-24.

25 Litvan I, Maclntyre A, Goetz CG, et al. Accuracy of the clinical diagnoses of Lewy body disease, Parkinson's disease, and dementia with Lewy bodies. Arch Neurol 1998;55:969-78.

26 Gelb DJ, Oliver E, Gilman S. Diagnostic criteria for Parkinson's disease. Arch Neurol 1999:56:33-9.

27 Altman DG. Practical statistics for medical research. London: Chapman \& Hall/CRC, 1991:447.

28 Tiraboschi $\mathbf{P}$, Hansen LA, Alford $M$, et al. Cholinergic dysfunction in diseases with Lewy bodies. Neurology 2000;54:407-1 1.

29 Perry E, Curtis M, Dick DJ, et al. Cholinergic correlates of cognitive impairment in Parkinson's disease: comparisons with Alzheimer's disease. J Neurol Neurosurg Psychiatry 1985;48:413-21.

30 Samuel W, Caligiuri M, Galasko D, et al. Better cognitive and psychopathologic response to donepezil in patients prospectively diagnosed as dementia with Lewy bodies: a preliminary study. Int J Geriatr Psychiatry 2000;15:794-802. 


\section{CORRESPONDENCE}

\section{Mexiletine on segmental hyperhidrosis}

Ishibashi et al ${ }^{1}$ reported the excellent efficacy of mexiletine for the treatment of segmental hyperhidrosis in two patients (who had syringomyelia and cavernous haemangioma of the spinal cord, respectively). They presented the decrement in the patients' sweat rate by oral administration of mexiletine.

Previously we performed a clinical study focusing on sweating and identified $10 \mathrm{pa}$ tients with segmental hyperhidrosis among 30 patients with syringomyelia. We followed up the patients with hyperhidrosis for $1-10$ (mean 5.0) years. The amount of sweating did not change in any of them during the follow up period, ${ }^{2}$ although we did not perform a quantitative analysis. Consequently, we speculated that hyperhidrosis persists for at least a year. It is possible that the course of signs in the cases reported by Ishibashi et al were modified by the growth or activity of spinal cord lesions. We consider it imperative that these authors describe any spinal cord lesions and how they may have shifted. However, although they did not mention the duration and time courses of the improvement in their patients, we suppose that the duration of the follow up for each patient would not have exceeded several months, judging from how the authors described their experience. In addition, even though they did not test the effects of mexiletine on control subjects or on other parts of the body in the same patients, we can be assured that the improvement in hyperhidrosis was due to the oral administration of mexiletine, on the assumption that the spinal cord tumour could not have changed in such a short time. We consider that it would be informative for clinicians if Ishibashi et al were to disclose the drug dosage and the time course of its effects and to describe the features of the spinal cord lesions.

K Sudo, Y Miyazaki, Y Tajima, A Matsumoto

Department of Neurology, Sapporo City Genera Hospital, Kita 11, Nishi 13, Chuo-Ku, Sapporo, 060-8604 Japan

K Tashiro, F Moriwaka

Department of Neurology, Hokkaido University School of Medicine, Sapporo, 060-8648, Japan

Correspondence to: Dr K Sudo sudo@med.hokudai.ac.jp

\section{References}

1 Ishibashi S, Yokota T, Inaba A, et al. Mexiletine is effective on segmental hyperhidrosis: report of two cases. J Neurol Neurosurg Psychiatry 2002;72:122.

2 Sudo K, Fujiki N, Tsuji S, et al. Focal (segmental) dyshidrosis in syringomyelia. $J$ Neurol Neurosurg Psychiatry 1999;67:106-8.

\section{Authors' reply}

We are grateful Sudo et al, as they allow us to clarify a point of our study that was not discussed in the paper recently published in this Journal. ${ }^{1}$ They asked about the possibility of natural remission and the non-specific effect of mexiletine on sweating.
We administered $200 \mathrm{mg} /$ day mexiletine or $400 \mathrm{mg} /$ day carbamazepine to our patients. Both patients noticed their hyperhidrosis was relieved within two days after administration. Although we did not perform a quantitative analysis several months after treatment, the clinical improvement of hyperhidrosis persisted. In addition, the magnetic resonance images of spinal cord lesions (syringomyelia and cavernous haemangioma) in both patients were followed up for two years. During the follow up period, the spinal cord lesions did not change their size, position, and intensity on magnetic resonance imaging. Therefore, the natural course of the spinal cord lesions could not explain the improvement of hyperhidrosis during the treatment and quantitative analysis in our patients.

The sweat rate of the area of observed hyperhidrosis was decreased without a change of the absolute sweat rate on the healthy side after oral administration of mexiletine. We calculated the ratio of the sweat rate on the affected side to that on the healthy side - the ratio was 2.13 before treatment and decreased to 0.97 on day 7 after the treatment. We therefore consider that the mexiletine had an excellent effect only on the area with hyperhidrosis. Although we did no test the effects of mexiletine on contro subjects, we think that the result on a healthy area of each patient was an appropriate internal controls for the evaluation of the drug's effect on hyperhidrosis

S Ishibashi, T Yokota Department of Neurology and Neurological Science, Graduate School of Medicine, Tokyo Medical and Dental University, 1-5-45, Yushima, Bunkyo-ku, Tokyo $113-8519$, Japan Correspondence to: $\mathrm{Dr} T$ Yokota tak-yokota.nuro@tmd.ac.jp

\section{Reference}

1 Ishibashi S, Yokota T, Shiojiri T, et al. Mexiletine is effective on segmental hyperhidrosis: report of two cases. J Neurol Neurosurg Psychiatry 2002;72:122

\section{Patent foramen ovale, cerebrovascular risk, and complement}

Nedeltchev et al $^{1}$ report that the presence of a patent foramen ovale (PFO) is a significant risk factor for recurrent cerebrovascular events, the risk being higher in patients with more than one previous embolic event. They highlight the absence of a current proven medical treatment or prevention regimen. Cardiac right to left shunting is present in a quarter of the population. It is thus worth drawing attention to a particular subgroup of patients with PFO that may be at an even more increased risk than the authors reportsport divers, most of whom fall within the age range of the above study.

Neurological sequelae constitute $80 \%$ of decompression sickness. Not only has neuroimaging shown an increased frequency of brain ischaemic lesions in divers, but also multiple such ischaemic lesions have been found specifically in sport divers with PFO. ${ }^{2}$ While PFO patency of haemodynamic significance is a risk factor that necessitates habit modification, often the radiological lesions do not correspond well to the neurological deficits of experienced divers.

This point, coupled with the increased risk of arterialisation of venous bubbles and the paradoxical nature of bubble genesis, suggest that a PFO is a risk factor in this subgroup for the development of neurovascular disease. ${ }^{3}$ Unknown is the added risk with age that remains to former divers. A poorly understood mechanism of bubble induced complement involvement in the pathogenesis of the neurological sequelae in decompression sickness has been suggested. ${ }^{4}$ Similarity of such symptoms to the postcoronary bypass syndrome lends support (and hope?) to complement based neuroprotective strategy options for the future.

\section{A K Demetriades}

The National Hospital for Neurology and Neurosurgery, Queen Square, London WCIN 3BG, UK

Correspondence to: $\operatorname{Dr}$ A K Demetriades; andreas.demetriades@doctors.org.uk

\section{References}

1 Nedeltchev K, Arnold M, Wahl A, et al. Outcome of patients with cryptogenic stroke and patent foramen ovale. $J$ Neurol

Neurosurg Psychiatry 2002;72:347-50.

2 Knauth M, Ries S, Pohimann S, et al. Cohort study of multiple brain lesions in sport divers: role of a patent foramen ovale. BM 1997;314:701-5.

3 Schwerzmann M, Seiler C, Lipp E, et al Relation between directly detected paten foramen ovale and ischemic brain lesions in sport divers. Ann Intern Med 2001;134:21-4.

4 Ward CA, McCullough D, Fraser WD Relation between complement activation and susceptibility to decompression sickness. J Appl Physiol 1987;62:1160-6.

5 D'Ambrosio AL, Pinsky DJ, Connolly ES. The role of the complement cascade in ischemia/reperfusion injury: implications for neuroprotection. Mol Med 2001;7:367-82.

\section{Authors' reply}

We thank Dr Demetriades for his comments on our study. While the average person with a patent foramen ovale (PFO) may not be at increased risk for neurological events, there seem to be subgroups of patients at increased risk. PFOs with large diameters, right to left shunting at rest, or high membrane mobility and PFOs associated with atrial septal aneurysms have been identified as "dangerous PFOs" by several investigators. ${ }^{1-3}$ In addition, coagulation abnormalities may promote paradoxical emboli in patients with PFO. ${ }^{4}$ To this list, Dr Demetriades adds special occupations or sports that may be dangerous in people with PFOs, specifically divers. Playing wind instruments has also been mentioned previously. ${ }^{5}$

However, many problems related to PFO remain unresolved. Even in groups that are believed to be at high risk for neurological events, deciding whether and how to treat a PFO cannot be derived from evidence based medicine. Deciding how to proceed depends on the opinion of the attending physician and is not based on data from randomised studies.

The PICSS (PFO in cryptogenic stroke study) showed that secondary prevention of cryptogenic stroke in patients with PFO by using warfarin or aspirin does not result in any difference. ${ }^{6}$ The PC-trial is an ongoing randomised trial we initiated to compare 
endovascular PFO closure versus medical treatment alone. We hope that it will provide useful information on secondary stroke prevention in patients with presumed paradoxical embolism. It is also conceivable that divers who have ever had "the bends" would benefit from PFO closure.

Recently reported data suggest links between decompression illness, migraine with aura, and right to left shunts. ${ }^{8}$ These observations not only extend the clinical manifestations of PFO but also bring into discussion new pathophysiological aspects of migraine. If the association between complicated migraine and PFO can be corroborated, randomised trial on $\mathrm{PFO}$ in such patients may be worth while.

K Nedeltchev, M Arnold, H Mattle

Department of Neurology, Inselspital, University of Bern, 3010 Bern, Switzerland

S Windecker, A Wahl

Department of Cardiology

Correspondence to: Prof Dr H P Mattle, Department of Neurology, Inselspital, CH-3010 Bern, Switzerland; heinrich.mattle@insel.ch

\section{Reference}

1 Schuchlenz HW, Weihs W, Horner S, et al. The association between the diameter of a patent foramen ovale and the risk of embolic cerebrovascular events. Am J Med

2000:109:456-62.

2 De Castro S, Cartoni D, Fiorelli $M$, et al. Morphological and functional characteristics of patent foramen ovale and their embolic implications. Stroke 2000;31:2407-13.

3 Mas JL, Arquizan C, Lamy C, et al. Patent Foramen Ovale and Atrial Septal Aneurysm Study Group. Recurrent cerebrovascular events associated with patent foramen ovale, atrial septal aneurysm, or both. N Engl J Med 2001:345:1740-6.

4 Chaturvedi S. Coagulation abnormalities in adults with cryptogenic stroke and patent foramen ovale. J Neurol Sci

1998; 160: 158-60.

5 Evers S, Henningsen $\mathrm{H}$, Ringelstein EB. Transient ischemic attacks caused by trumpet playing. Neurology 1998;51:1709-10.

6 Homma S, Sacco RL, Di Tullio MR, et al. PFO in Cryptogenic Stroke Study (PICSS) Investigators. Effect of medical treatment in stroke patients with patent foramen ovale: patent foramen ovale in cryptogenic stroke study. Circulation 2002; 105:2625-31.

7 AGA Medical Corporation. PC-trial: patent foramen ovale and cryptogenic stroke. 18 January 2002. http://www.amplatzer.com/ physicians/pfo/pctrial/.

8 Wilmshurst P, Nightingale S. Relationship between migraine and cardiac and pulmonary right-to-left shunts. Clin Sci (Lond) 2001;100:215-20.

\section{Demyelination in the brain as a paraneoplastic disorder: candidates include some cases of seminoma and central nervous system lymphoma}

We read with interest the report of AyusoPeralta et al, ${ }^{1}$ which describes a 58 year old woman who presented with several neurological symptoms. Brain imaging was consistent with leukoencephalopathy, and analysis of blood and cerebral spinal fluid was unrevealing. A few months later the patient experienced further neurological deterioration and an open brain biopsy showed central nervous system (CNS) lymphoma, together with diffuse demyelination.

The authors observed that the presentation of cerebral lymphoma as a diffuse leukoencephalopathy is not frequent and they discuss possible aetiologies of the predominant demyelination in their case. They do not mention the possibility of a paraneoplastic aetiology.

The authors reference a similar case ${ }^{2}$ previously reported in the Journal. That report also does not acknowledge a possible paraneoplastic aetiology for prominent diffuse brain demyelination preceding the discovery of CNS lymphoma. Two other recent reports in the Journal ${ }^{3-4}$ described focal tumour-like lesions of brain demyelination that preceded the discovery of CNS lymphoma. Only one of these reports ${ }^{4}$ mentioned laboratory data that suggested consideration of a paraneoplastic aetiology, the presence of serum antibodies directed against myelin oligodendrocyte glycoprotein.

One report elsewhere $\mathrm{s}^{5}$ has described a patient who had a non-neurological malignancy and seminoma and who subsequently developed a paraneoplastic syndrome simulating encephalitis associated at autopsy with multiple foci of demyelination confined to cerebral white matter. Two other reports elsewhere ${ }^{6-7}$ have described biopsy confirmation of large focal demyelinating lesions in the brain associated with seminoma. The authors of these three reports all strongly considered the possibility of a paraneoplastic aetiology for the brain demyelination associated with seminoma, probably because the temporal association was close and the spatial association was distant.

The associations between brain demyelination and CNS lymphoma have been close both temporally and spatially, making considerations of aetiology more complex. Taken together, the seminoma reports and the CNS lymphoma reports have many similarities in their patterns of associated brain demyelination, raising the possibility of similar mechanisms of demyelination. Many questions concerning aetiology remain unanswered. Given the information available, we suspect a paraneoplastic aetiology in all of these cases We feel that future reports of brain demyelination associated with CNS lymphoma should consider this possibility in their data collection and in their discussion of results.

J H Jaster

Delta Medical Center, 1905 Harbert Avenue Memphis, Tennessee 38104, USA

F C Dohan Jr

Division of Neuropathology, Department of Pathology, University of Tennessee, Memphis, Memphis, Tennessee 38163, USA

T F O'Brien

Department of Pathology, Methodist University Hospital 1265 Union Avenue, Memphis, Tennessee 38104, USA

Correspondence to: Dr J H Jaster, Department of Medicine, University of Tennessee, 1905 Harbert Avenue, Memphis, Tennessee 38104, USA harbert38104@yahoo.com

\section{References}

1 Ayuso-Peralta L, Orti-Pareja $M$, Zurdo-Hernandes $M$, et al. Cerebra lymphoma presenting as a eukoencephalopathy. J Neurol Neurosurg Psychiatry 2001;71:243-6.

2 Brecher K, Hochberg FH, Louis DN, et al Case report of unusual leukoencephalopathy preceding primary CNS lymphoma. J Neurol Neurosurg Psychiatry 1998;65:917-20.

3 Alderson L, Fetell MR, Sisti M, et al. Sentinel lesions of primary CNS lymphoma. J Neurol Neurosurg Psychiatry 1996;60:102-5.

4 Kuhlmann T, Schroter A, Dechent $P$, et al. Diagnosis of a multifocal $B$ cell lymphoma with preceding demyelinating central nervous system lesions by single voxel proton MR spectroscopy. J Neurol Neurosurg Psychiatry 2001;70:259-62.
5 Kaluza J, Slowinski J, Buiny T, et al. Paraneoplastic syndrome simulating encephalitis in the course of testicula seminoma. Folia Neuropathol 1997;35:24-8.

6 Wong K, Poon P, Berry K, et al. Paraneoplastic demyelinating disorder in the brain of a patient with seminoma. J Comput Assist Tomogr 1998;22:136-8.

7 Jaster JH, Bertorini TE, Dohan FC, et al. Solitary focal demyelination in the brain as a paraneoplastic disorder. Med Pediatr Oncol 1996;26: $111-5$

\section{Genotype predisposition to leukoaraiosis}

Leukoaraiosis, which can cause symptoms ranging from a mild cognitive impairment to severe subcortical dementia, ${ }^{1}$ is a significant public health problem. One quarter of subjects aged 65 years or over are affected by some degree of white matter changes. ${ }^{2}$ Ischaemic demyelination and small vessel disease seem to be important features of the underlying pathological process of this entity. ${ }^{2}$ Age, hypertension, and a previous stroke event have been proved to be the main clinical risk factors. ${ }^{12}$ A number of genetic susceptibility factors for leukoaraiosis have been put forward, with the assumption of polygenic aetiological factors. We were pleased to read the article by Hassan et al in this journal. ${ }^{3}$ The authors stated that the angiotensin converting enzyme insertion/deletion (ACE I/D) polymorphism D/D genotype was a significant independent predictor for leukoaraiosis in patients presenting with classic lacunar syndomes. ${ }^{3}$ We earlier conducted large prospective studies in which we also examined the importance of the ACE D allele and other common mutations in the development of small vessel infarction and leukoaraiosis. ${ }^{4}$ Our results were consistent with the findings of Hassan et al and support their results from several other aspects. (1) Our stoke study confirmed the genetic heterogeneity of ischaemic stroke in that the ACE D/D genotype proved a significant susceptibility genotype for small vessel brain infarction, as did the Leiden $\mathrm{V}$ mutation for large brain infarction. ${ }^{4}(2)$ In our leukoaraiosis study, the ACE D/D genotype was found to be a significant risk factor for leukoaraiosis in combination with brain infarction. ${ }^{5}$ (3) We also reported that clustering of the homozygous MTHFR 677TT and ACE D/D mutations in one person can mean a moderate (about fivefold risk), but highly significant $(p<0.0005)$ risk of leukoaraiosis without infarction. ${ }^{5}$ These data from different approaches reconfirm the possible aetiological role of the ACE D/D genotype in leukoaraiosis relating to small vessel brain disease. The genotype differences may explain why some patients who are exposed to clinical risk factors such as hypertension, exhibit a much higher susceptibility to leukoaraiosis than other subjects with the same clinical risk factors. Besides the classic clinical risk factors, the consistently growing knowledge of the genetic background of leukoaraiosis may permit the recognition of a large population at high risk of a new type of white matter damage, and hence this may lead to a more effective prevention.

Z Szolnoki, M Szabó Department of Neurology and Neurophysiology, Pándy Kálmán County Hospital, Gyula, Hungary

F Somogyvári

Central Laboratory, Pándy Kálmán County Hospital

Correspondence to: Dr Z Szolnoki, H-5600 Békéscaba, Pipacs köz 9, Hungary; szolnoki99@hotmail.com

\section{References}

1 Pantoni L, Garcia JH. Pathogenesis of

leukoaraiosis, a review. Stroke 1997;28:652-9. 
2 van Giin J. Leukoaraiosis and vascular dementia. Neurology 1998;51:3-8.

3 Hassan A, Lansbury A, Catto A, et al. Angiotensin converting enzyme insertion/deletion genotype is associated with leukoaraiosis in lacunar syndromes. J Neurol Neurosurg Psychiatry 2002;72:343-6.

4 Szolnoki Z, Somogyvári F, Kondacs A, et al. Evaluation of the roles of the Leiden $V$ mutation and ACE I/D polymorphism in subtypes of ischaemic stroke. J Neurol subtypes of ischaem $2001 ; 248: 756-61$.

5 Szolnoki Z, Somogyvari F, Kondacs A, et al. Evaluation of the roles of common genetic mutations in leukoaraiosis. Acta Neurol Scand $2001 ; 104: 281-7$.

\section{BOOK REVIEWS}

\section{Neurochemistry of consciousness: neurotransmitters in mind}

Edited by Elaine Perry, Heather Ashton, and Allan Young (Pp 346, EUR65.00). Published by John Benjamins Publishing Company, Amsterdam, 2002. ISBN 90-272-5156-8

Consciousness is a portmanteau word, full of rich and different meanings: contrast Marxian, Freudian, and anaesthesiologists' use of the term. In recent years it has also become a fashionable hunting ground for neuroscientists, who are rarely troubled by such complexities. For them, consciousness is being awake rather than asleep, being reducible to awareness. Sweeping aside centuries of philosophical debate, they ponder over whether "it" "resides" in specific anatomical brain structures, in microtubules, in patterns of neurotransmitter release, or whatever. The present book is typical of this type of cheerfully unsophisticated empiricism: its hunt for what the editors call "NCCs"-neura correlates of consciousness-focuses on neurotransmitters, hence the subtitle. However, the concern with "mind" ceases at that point; this elusive phenomenon finds no place in the book's index. The central question for the editors seems to be whether the acetylcholine or the dopaminergic system is the more likely substrate for conscious awareness. This reduc tionism characterises most of the chapters. That on memory, for instance, abandons even animal memory for a discussion of a physio logical phenomenon called long term poten tiation, and even the psychoanalyst Mark Solms, on dreams, who surely ought to have a broader perspective, confines himself to contrasting cholinergic and dopaminergic hypotheses. However, the authors are clearly writing to an editorial brief: each chapter, in a book ranging from discussions of attention and motivation through psychotropic drug mechanisms to mental retardation and autism, following a brief nod to marginally wider concerns, offers a neurotransmitter by neurotransmitter list of potential association or correlations with "states of awareness." Within these limitations many of the chapters provide competent student friendly overviews of their themes. If the book's pretensions weren't so much larger this would be fine; as it is, those hoping for a more multilevel or theoretically informed discussion will be disappointed.

\section{Risk control and quality management in neurosurgery}

Edited by $\mathrm{H}-\mathrm{J}$ Steiger and E Uhl (Pp 227 EUR 108.000). Published by Springer Verlag Wien, Wien, 2001. ISBN 3-211-83678-0

This is an interesting and timely publication. The book contains a compilation of material presented at an international meeting held in October 2002. It has been divided into various sections that take the reader through grouped papers and topics and finally a projection into the future. As would be expected, the material covers experience and lessons gained in other areas such as aviation and nuclear research. The authors, generally senior in status, originate from Europe, the United States, and the United Kingdom and therefore offer a diverse collection of views, opinions, and experience relevant to a very wide readership. The increasing requirements for quality assessment and competency make this a very valuable reference book for both departmental and institutional libraries. However, it certainly will be of value to individual readers. It should be recommended reading for trainees to understand the principles and the ongoing thought behind many of the practices and control measures that they will encounter and will need to participate in as their experience and seniority advance. The quality of contributions and the outline of the information do vary, as would be expected in such a compilation, but overall very few pages or chapters do not prove insightful nor provide useful information and guidelines. It will be of value to all medica disciplines, since the principles are universal and the terms of reference or yardsticks used are convertible or transferable. It is highly recommended.

J Van Dellen

\section{Primary progressive multiple sclerosis}

Edited by M Filippi and G Comi (Pp 128 EUR47.95). Published by Springer, Milan, 2002. ISBN 88-470-0167-6

The field of multiple sclerosis (MS) is awash with literature on every aspect of the disease ranging from epidemiology and genetics to pathology and treatments. It is unusual, therefore, to find a lacuna in this niche but this book seems to have found one.

Primary progressive multiple sclerosis is written to encapsulate the latest evidence on aspects of this condition, which until recently was not regarded as important in understanding demyelinating disease. Filippi and Como have brought together all the important players in the study of primary progres sive MS. Their contributions summarise the latest information on the epidemiology, genetics, immunology, pathology, imaging, and clinical trials and therapies in primary progressive MS. This book is meant to be a useful guide to the subject and does not profess to be an authoritative account. However, it occasionally is a little too brief in its explanations and definitely lacks pictures, tables, and diagrams in the early part of the book. This makes it a rather bland and dry account initially. When the diagrams and scanned images do appear in the latter parts of the book, many of them lack definition and it is not always easy to see the details that are being referred to.
All in all this is a good up to date summary of the latest news in primary progressive MS and in particular the imaging aspects of the disease, as would be expected from the interests of the editors. It would be a useful adjunct to other literature for those working in the field of demyelinating disease.

Omar Malik

\section{Multiple sclerosis: a guide for the newly diagnosed, 2 nd edn}

By N J Holland, T J Murray, and S C Reingold (Pp 160, US\$21.95). Published by Demos Medical Publishing Inc, New York, 2002. ISBN 1-888799-60-9

This book is an invaluable guide for patients with multiple sclerosis (MS), as well as their friends and families. The fact that a second edition has become necessary is extremely encouraging for those involved with MS and highlights the recent therapeutic advances for this still devastating diagnosis. Most people who develop MS are desperate for information about their new disease and many turn to the internet to find this. Unfortunately, they are then faced with misleading or simply incorrect information, which can leave patients confused or disillusioned.

The authors present detailed information in the first two chapters covering the pathological processes causing the symptoms of MS and the diagnostic tests in use. Uncertainties in both these fields are explained. The next two chapters deal with treatments, including conventional and alternative or complementary therapies; the text is clear about the lack of a cure for MS but discusses all the options including steroids for acute attacks, disease modifying drugs, and symptomatic treatments. There is a whole chapter on the important issues of lifestyle-diet, rest, sexual function, pregnancy, etc-that help patients to control their condition. A further chapter concentrates on the psychological impact of a diagnosis of MS and its effect on relationships. Employment issues are deservedly dealt with on their own, with practical advice on when and how to disclose the diagnosis and the legal implications of disclosure both at work and on application forms such as those for health and life insurance.

The latter part of the book deals with clinical and research trials in MS that will help patients to understand how trials are designed and why treatments are offered to patients with specific disease types. The many fields in which MS research is ongoing are described and the questions being asked by investigators are well presented.

The book ends with more practical advice on how to get further information about specific topics; however, this is predominantly aimed at the North American readership with emphasis on the MS societies of the United States and Canada.

In summary, this is an excellent book, which presents all the facts in a straightforward but sympathetic way. As well as the medical facts about the disease, it is full of practical advice covering all life topics, areas that are often neglected by busy physicians. It is highly recommended to all those whose lives have been affected by this disease.

Valerie Stevenson 
Disordered mind and brain: the neural basis of mental symptoms

By Peter F Liddle (Pp 320, £40.00). Published by Gaskell, London, 2001. ISBN 1-90124265-X

The premise of this book is that the key to understanding the neural basis of the major mental disorders is an understanding of the origin of five symptom clusters or dimensions common to these disorders. These are reality distortion (hallucinations and delusions); disorganisation (of thought and behaviour); psychomotor poverty and excitation; depression and elation; and anxiety. Thus, there are five chapters each devoted to a description of a specific dimension and an exposition of how it is correlated with cognitive abnormalities derived from the dysfunction of specific neural processes.

These central chapters are preceded by five chapters describing the neuroscience of brain systems thought to be involved in generating the various symptom clusters. These are brief and the literature reviews are in no way comprehensive. Nevertheless, they serve the purpose of informing the reader of the basic neuroanatomical and neurophysiological concepts that underpin Professor Liddle's approach to understanding mental illness.

The final four chapters summarise the current evidence regarding the neurobiology of schizophrenia, bipolar affective disorder, obsessive compulsive disorder, and psychopathy.
Each ends with a synthesis that integrates this with the previous account of how the symptom clusters arise.

The explanatory power of Professor Liddle's thesis concerning the neural basis of mental symptoms is stronger for some symptom dimensions, such as reality distortion, than others, such as distortion. But it is the general unifying approach that is the major strength of this book-the detail will certainly be honed over the next decade. Another strength is that this is a self contained book! It assumes no neuroscientific or medical knowledge other than the most basic. There are many excellent colour illustrations. Therefore, this book can be highly recommended to anybody interested in the disordered mind and brain.

Eileen Joyce

\section{CORRECTIONS}

Rosso SM, van Swieten JC, Roks G, et al. Apoliproprotein E4 in the temporal variant of frontotemporal dementia. $J$ Neurol Neurosurg Psychiatry 2002;72:820.

Due to the style used in house for listing authors affiliations in the Letters section of the journal, the author's names have been incorrectly listed. The correct order should read as follows:

Rosso SM, Roks G, Cruts M, van Broeckhoven C, Heutink P, van Duijn CM, van Swieten JC
This also applies to:

Lünemann JD, Kassim N, Zschenderlein $\mathrm{R}$, et al. Rhabdomyolysis during interferon- $\beta$ la treatment. J Neurol Neurosurg Psychiatry 2002;2:274.

The correct order of the authors is: Lünemann JD, Schwarzenberger B, Kassim N, Zschenderlein R, Zipp F.

Aarsland $\mathbf{D}$ et al. Donepezil for cognitive impairment in Parkinson's disease: a randomised controlled study. J Neurol Neurosurg Psychiatry 2002;72:708-12. An error occurred in the production process in which the codes of the two lines were erroneously interchanged. The correct figure appears below:

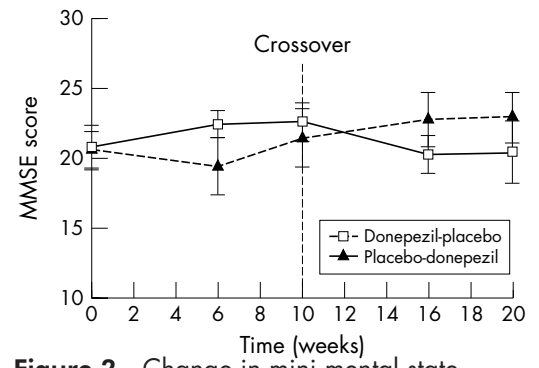

Figure 2 Change in mini mental state examination (MMSE) score from baseline over the two treatment sequences. Values are mean (SE). 